Scaling properties of coating flows in rectangular channels

de Lozar, A. and Hazel, A.L. and Juel, A.

2007

MIMS EPrint: 2007.233

Manchester Institute for Mathematical Sciences

School of Mathematics

The University of Manchester

\footnotetext{
Reports available from: http://eprints.maths.manchester.ac.uk/

And by contacting: The MIMS Secretary

School of Mathematics

The University of Manchester

Manchester, M13 9PL, UK
} 


\title{
Scaling Properties of Coating Flows in Rectangular Channels
}

\author{
A. de Lózar, A. L. Hazel, and A. Juel \\ Manchester Centre for Nonlinear Dynamics and School of Mathematics, University of Manchester, \\ Oxford Road, Manchester M13 9PL, United Kingdom \\ (Received 2 March 2007; published 3 December 2007)
}

\begin{abstract}
We present an experimental study of the aspect-ratio dependence of two-phase displacement flows in channels of rectangular cross section. Above a buoyancy-dependent threshold $\widehat{C a}_{t}$, we find that the bulk features of the flow depend only on a modified capillary number $\widehat{\mathrm{Ca}}=[1+0.12(\alpha-1)+0.018(\alpha-$ $\left.1)^{2}\right] \mathrm{Ca}$, where $\mathrm{Ca}$ is the unmodified capillary number and $\alpha$ is the aspect ratio. This novel scaling has tremendous practical significance because it implies that the bulk features of the flows in any rectangular channel can be inferred from those in a square channel for $\widehat{\mathrm{Ca}}>\widehat{\mathrm{Ca}}_{t}$.
\end{abstract}

DOI: 10.1103/PhysRevLett.99.234501

The displacement of a liquid by an air finger is a generic two-phase flow that underpins applications as diverse as microfluidics, thin-film coating, enhanced oil recovery, flows in porous media, manufacture of catalytic converters [1], gas-assisted manufacture of plastic moulds [2], and biomechanics of the lungs [3]. When a large volume of air is driven through a tube initially filled with a viscous liquid, after a short distance, the air forms a single finger that advances at a constant speed. The finger is surrounded by a liquid film whose thickness increases monotonically with the capillary number, $\mathrm{Ca}=\mu U_{f} / \sigma$, the ratio of viscous to surface-tension forces; $\mu$ is the viscosity of the liquid, $U_{f}$ is the velocity of the finger tip, and $\sigma$ is the interfacial tension. These interfacial flows were originally studied in tubes of circular cross section [4-6], but circular tubes are rarely encountered in applications [7]. Thus, flows in rectangular tubes have been proposed as a paradigm for flows in nonaxisymmetric geometries. The majority of previous studies in rectangular geometries, however, have been limited to flows in square or nearsquare tubes $[1,8]$, or in quasi-two-dimensional channels (Hele-Shaw cells) $[9,10]$. Those few studies at intermediate aspect ratios considered the buoyancy-driven "free" rise of bubbles [11], rather than the more general driven-flow problem. In this Letter, we examine the dependence on the aspect ratio, $\alpha=w / b$ (where $w$ and $b$ are the width and depth of the channel, respectively), of driven twophase displacement flows in rectangular channels. Our experimental results indicate that the bulk features of these flows have a simple dependence on $\alpha$. This study has a direct impact on the field of microfluidic devices, where rectangular microchannels are routinely manufactured with moderate aspect ratios $1 \leq \alpha \leq 10$ [12]. The choice of the microchannel aspect ratio is important, because the channel width is often used to control the local flow velocity, the channel depth and flow rate being kept constant.

In a Hele-Shaw cell, the development of the advancing finger is known as the Saffman-Taylor instability and is an
PACS numbers: 47.85.mb, 47.15.gp, 47.20.Ma, 47.55.N-

archetype of front-propagating, pattern-forming systems. In this geometry, the flow can be modeled $[9,13]$ in two dimensions (2D) by using the depth-averaged NavierStokes equations, which support steadily propagating single-finger solutions governed by one parameter: $1 / B=$ $12 \alpha^{2} \mathrm{Ca}$. Experimental studies $[10,14]$ measured the ratio of the finger width $L$ to the width of the channel $w, \lambda=$ $L / w$, as a function of $1 / B$. Quantitative agreement with theory was found for moderate values of $1 / B$ and $\alpha \geq 20$ by including the effect of the deposited fluid film in an effective surface tension [15]. For very large values of $\alpha$, however, $\lambda$ depends on $\mathrm{Ca}$ and $\alpha$ separately, rather than the combined parameter $1 / B$ [16]. In square and near-square tubes, depth averaging is not appropriate and the natural governing parameter is the unmodified capillary number $\mathrm{Ca}$; but an analytical description of the flow above the asymptotic limit of small $\mathrm{Ca}$ remains elusive $[17,18]$. In these cases $\lambda$, a 2D measure, no longer captures the threedimensional nature of the flow. A suitable bulk measure is the wet fraction $m=1-Q /\left(A U_{f}\right)$ : the ratio of the liquid volume that remains once the finger has exited the channel to the total volume of the channel; here, $Q$ is the flow rate of the liquid and $A$ the cross-sectional area of the channel. In this Letter we uncover a novel scaling that connects the bulk features of the flow, quantified by the wet fraction, between the two geometric limits of the square channel and the Hele-Shaw cell.

Our channel consisted of two $60 \mathrm{~cm}$ long float-glass plates separated by two precision-machined stainless steel spacers. The use of movable spacers, connected to a translation stage, allowed us to study rectangular channels with aspect ratios ranging from $\alpha=1$ to 15 . The silicone oil that initially filled the channel was withdrawn at a constant flow rate $Q$, using a syringe pump. Air at atmospheric pressure penetrated the liquid and formed a single finger with a constant tip velocity $U_{f}$ after initial transients had subsided. $U_{f}$ was determined by analyzing movies of the advancing finger recorded with a top view camera, whose maximum resolution was 31 pixels $/ \mathrm{mm}$. We used three 
grades of silicone oil, which fully wet the channel and whose physical properties are listed in [19]. By varying the viscosity of the liquid over three orders of magnitude, four decades of capillary number could be explored $(1 \times$ $10^{-4}<\mathrm{Ca}<3$ ).

The channel width was also measured by computer analysis of pictures taken with the camera. The accuracy of the width measurement determines the precision with which $m$ and $\alpha$ can be obtained, and so a relatively large channel depth, $b=3.00 \mathrm{~mm}$, was chosen, in order to ensure an error lower than $1 \%$ in the determination of the wet fraction for the square channel, in which the width is minimum, $w=3.00 \mathrm{~mm}$. The plates were adjusted until the difference between three width measurements, taken at distances 15,30 , and $45 \mathrm{~cm}$ from one end of the channel, was less than $0.02 \mathrm{~mm}$ (i.e., less than $0.6 \%$ of the width of the square channel). A consequence of this choice of $b$ is a non-negligible Bond number (ratio between buoyancy and capillary forces: $\mathrm{Bo}=\rho g b^{2} / 4 \sigma \simeq 1.0 ; \rho$ is the density of the liquid and $g$ the acceleration due to gravity), which is sufficient to suppress the Rayleigh-Plateau instability [20].

To test our setup, we first measured the wet fraction in a square channel as a function of the capillary number (see Fig. 1). The excellent collapse of the experimental data measured for all three grades of silicone oil indicates that inertial effects are negligible. This is not surprising because the Weber number, the ratio of inertial to capillary forces [21], was very low (We $\left.=\rho U_{f}^{2} b / 2 \sigma<0.02\right)$. We compare our data with the numerical results of Hazel and Heil [8] who simulated the system in the absence of gravity. Our measurements are in close agreement only at

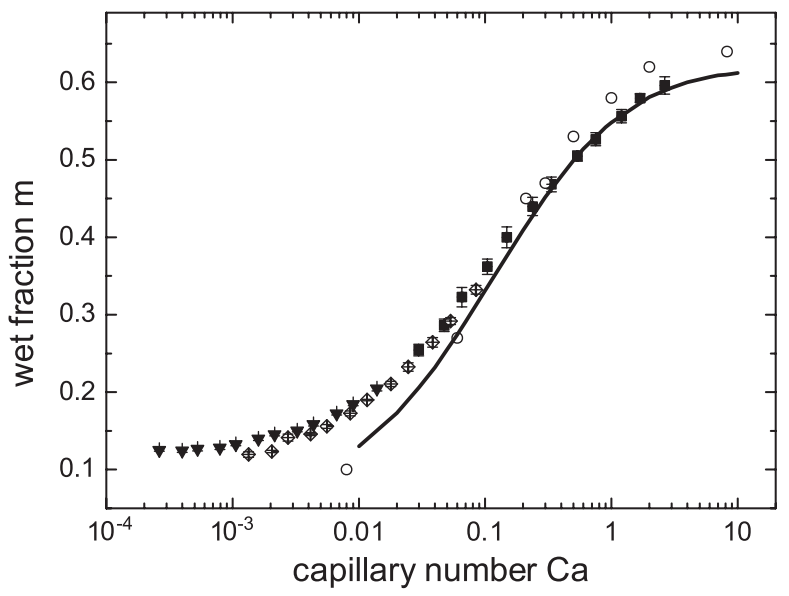

FIG. 1. Wet fraction in a square channel as a function of the capillary number. Each data point represents the average of five separate experiments; the standard deviation is indicated by the error bars. The experiments were performed with silicone oils of nominal dynamic viscosities $0.934 \mathrm{cP}(\boldsymbol{\nabla}), 4.815 \mathrm{cP}(\diamond)$ and $97.4 \mathrm{cP}(\boldsymbol{\square})$. The line is Hazel and Heil's zero-gravity numerical prediction [8] and the open circles represent the experimental results of Kolb and Cerro [1] in a vertical channel of depth $b=$ $2.0 \mathrm{~mm}$. high capillary numbers $(\mathrm{Ca}>0.25)$ when viscous forces dominate and the air finger is centered in the channel's cross section. As Ca decreases, buoyancy forces overwhelm the viscous forces, the air finger rises, and its cross-sectional area decreases, leading to an increase in the wet fraction compared to the zero-gravity simulations. In the regime where buoyancy can be neglected, our results are also consistent with the measurements of Kolb and Cerro [1] in a vertical channel of depth $b=2.0 \mathrm{~mm}$.

In Fig. 2 the relative finger width, $\lambda$, is shown as a function of the (Saffman-Taylor) parameter $1 / B$ for different values of $\alpha$. Each data point on the graph represents an average of several experiments and the small (often indistinguishable) error bars indicate the high level of reproducibility of the results. The relative finger widths measured at the aspect ratios $\alpha=8,10,12$, and 15 collapse almost perfectly onto a single curve, whereas at smaller aspect ratios $(\alpha \leq 6)$ the data do not superpose. Our results for $\alpha \geq 8$ compare favorably with the measurements performed by Tabeling et al. [14] at higher aspect ratios ( $\alpha=$ 20.3, 34, and 65), establishing $1 / B$ as the appropriate governing parameter for $\lambda$ when $\alpha \gtrsim 7$. The Bond number in their experiments was at least four times smaller $(\mathrm{Bo}<$ 0.25 ) than in our setup, which suggests that buoyancy forces do not significantly affect the finger width in highaspect-ratio channels. Our results are also consistent with the experiments of Clanet et al. [11], who found that the velocity of long, gravity-driven bubbles in rectangular tubes varies according to two different scaling laws depending on whether $\alpha \geq 7$ or $\alpha<7$.

Figure 3 shows the measured wet fractions as functions of the capillary number for the same set of experiments as presented in Fig. 2. At high $\mathrm{Ca}$ the wet fraction increases

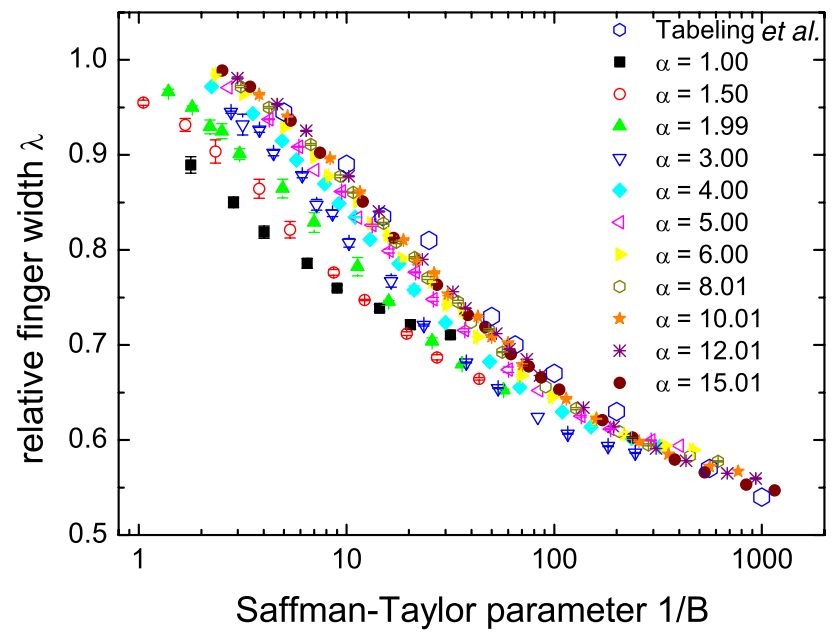

FIG. 2 (color online). Relative finger width as a function of the Saffman-Taylor parameter for 11 different values of the aspect ratio, $\alpha$, between $\alpha=1.00$ and $\alpha=15.01$. The open hexagons represent the experimental results of Tabeling et al. [14] at aspect ratios $\alpha=20.3,32.3$, and 65 . The results collapse onto a single line for aspect ratios $\alpha \geq 8$. 


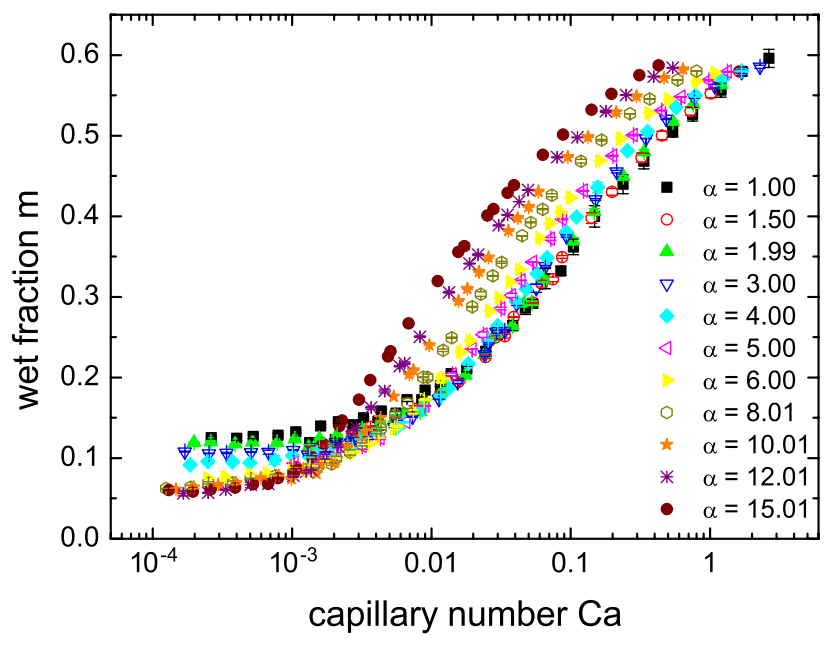

FIG. 3 (color online). Wet fraction as a function of the capillary number for 11 different values of the aspect ratio, $\alpha$. Note that the measurements for $\alpha=1.00, \alpha=1.50$, and $\alpha=1.99$ collapse for $\mathrm{Ca}>0.01$, indicating virtual independence of $\alpha$ in near-square channels.

with the aspect ratio, while at low Ca the opposite tendency is observed. This is important in liquid recovery applications because it implies that at high Ca more liquid can be recovered by using a narrow tube, but at low $\mathrm{Ca}$ a wide tube recovers the greatest relative volume of liquid. At the lower aspect ratios $(\alpha=1.00,1,50$, and 1.99), the points appear to lie on a single curve for $\mathrm{Ca}>0.01$, in agreement with the numerical results of Hazel and Heil [8], who found that the wet fraction in channels with $1 \leq \alpha \leq 2$ is independent of the aspect ratio, despite significant variations in the flow details.

As $\mathrm{Ca}$ approaches zero, the wet fraction approaches an aspect-ratio-dependent asymptotic value, the static wet fraction. We estimate the static wet fractions for each aspect ratio by taking the mean value of the four points corresponding to the lowest values of $\mathrm{Ca}$ in Fig. 3 (ranging over half an order of magnitude in Ca), see Fig. 4. The static wet fraction decreases monotonically with aspect ratio. Indeed, $m(\mathrm{Ca}=0)$ in a square channel is approximately twice that in a rectangular channel with $\alpha=15$. For comparison, we plot the theoretical prediction in the absence of buoyancy, calculated by analytically solving the Laplace-Young equation (see, e.g., $[17,18]$ ). The presence of a hydrostatic pressure gradient modifies the static interface shapes [22] and increases the wet fraction by a factor of approximately 2 to 3 . Yet, both the experimental and theoretical curves exhibit a similar variation with $\alpha$. For $\alpha \geq 8$, the experimentally measured wet fraction remains approximately constant, indicating that the system becomes independent of the aspect ratio in this limit in accordance with the Saffman-Taylor theory.

The similar curve shapes in Fig. 3 suggest the existence of a single control parameter, say $\widehat{\mathrm{Ca}}$. A balance between viscous and surface-tension forces in the liquid film gives

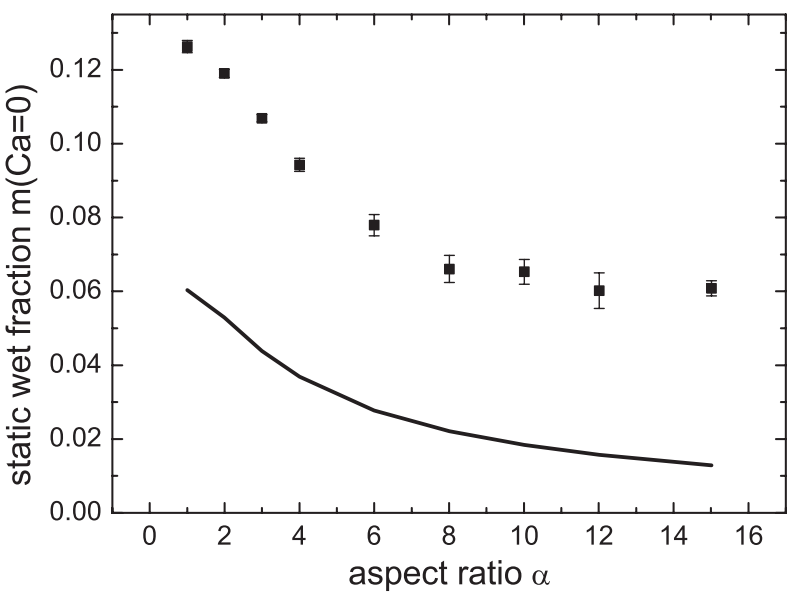

FIG. 4. Static wet fraction as a function of the aspect ratio, obtained by taking the mean value of the four points corresponding to the lowest values of $\mathrm{Ca}$ in Fig. 3 for each aspect ratio; the error bars represent the standard deviation of the four data points. The line is the theoretical prediction in absence of buoyancy (see, e.g., $[17,18]$ ).

$\mathrm{Ca}^{2} \mathbf{u}^{*} \sim \nabla \kappa$, where $\kappa$ is the sum of the two principal curvatures of the finger, and $\mathbf{u}^{*}=\mathbf{u} / U_{f}$ is the dimensionless liquid velocity. The capillary number naturally separates from the geometrical factors (derivatives and curvature) motivating a scaling of the form $\widehat{\mathrm{Ca}}=f(\alpha) \mathrm{Ca}$. Moreover, this form is appropriate in the square-channel limit $[f(\alpha)=1]$ and in Hele-Shaw channels. In the latter case, $w \gg b$ implies that $\nabla^{2} \sim 1 / b^{2}$ and assuming negligible vertical interface curvature gives $\kappa \sim 1 / w$. The result $f(\alpha)=\alpha^{2}$ then follows directly from the axial component of the force balance. We approximate the scaling function by a polynomial $f(\alpha)=1+a_{1}(\alpha-1)+a_{2}(\alpha-1)^{2}$, consistent with the two limits described above. Fitting a cubic polynomial to each curve in Fig. 3 and minimizing the sum of the squares of the differences between these curves yields $a_{1}=0.12 \pm 0.02$ and $a_{2}=0.018 \pm 0.001$ [23]. Order-of-magnitude estimates for the coefficients can be found by assuming that all three terms balance at a transitional aspect ratio $\alpha_{t}$, so $a_{1} \sim 1 /\left(\alpha_{t}-1\right)$ and $a_{2} \sim$ $1 /\left(\alpha_{t}-1\right)^{2}$. Clanet et al.'s [11] work and our $\lambda$ measurements suggest that $\alpha_{t} \sim 8$ and, indeed, minimizing the sum of the relative errors between the above estimates and numerical values gives $\alpha_{t} \sim 8.5$.

In Fig. 5 we plot the wet fraction as a function of the rescaled capillary number $\widehat{\mathrm{Ca}}$. All the data collapse almost perfectly over two orders of magnitude in $\widehat{\mathrm{Ca}}$ above a threshold value, $\widehat{\mathrm{Ca}}_{t}=0.035$. The threshold is chosen to be the lowest value of $\widehat{\mathrm{Ca}}$ for which the wet fractions for all values of $\alpha$ differ by less than $2 \%$, i.e., they remain equal to within our experimental error $( \pm 1 \%)$. For $\widehat{\mathrm{Ca}}<\widehat{\mathrm{Ca}_{t}}$, the points separate with a monotonic dependence on the aspect ratio. For high $\widehat{\mathrm{Ca}}$ the wet fraction approaches a buoyancy- 


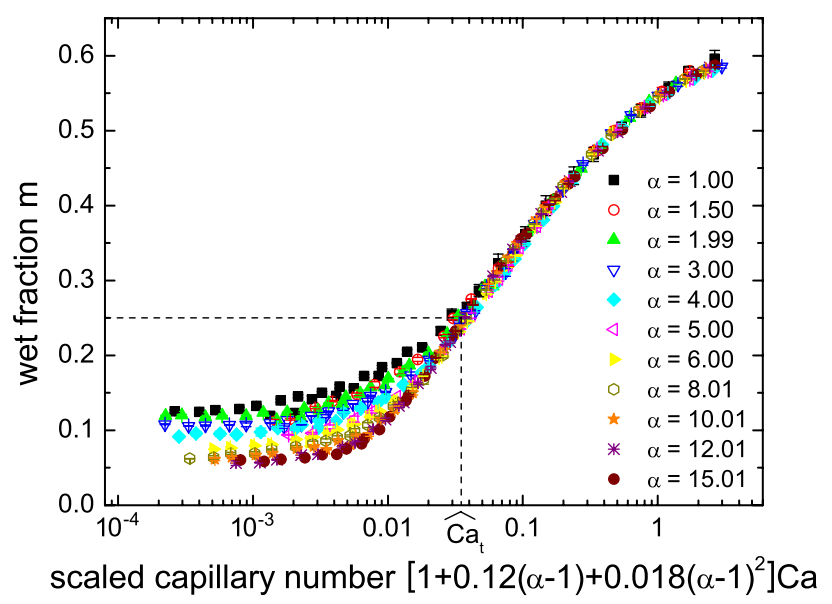

FIG. 5 (color online). Wet fraction as a function of the scaled capillary number for 11 different values of the aspect ratio, $\alpha$. Above a threshold value $\widehat{\mathrm{Ca}}_{t}=0.035$, the wet fraction depends solely on a new modified capillary number, $\widehat{\mathrm{Ca}}=[1+0.12(\alpha-$ 1) $\left.+0.018(\alpha-1)^{2}\right] \mathrm{Ca}$.

independent asymptotic limit [8] and so we expect the scaling to be valid beyond the measured upper limit of $\widehat{\mathrm{Ca}}$. We also expect that the scaling will apply at lower Bond numbers (channels of smaller depth or a liquid of higher surface tension), but the threshold $\widehat{\mathrm{Ca}}_{t}$ at which the scaling breaks down is likely to be dependent on buoyancy, which dominates at low $\widehat{\mathrm{Ca}}$. We speculate that $\widehat{\mathrm{Ca}}_{t}$ would decrease in the absence of buoyancy. $\widehat{\mathrm{Ca}}_{t}$ will remain nonzero, however, because $m$ always depends on $\alpha$ in the static limit, irrespective of the buoyancy forces, see Fig. 4.

In conclusion, we have shown that the scaling parameter governing the Saffman-Taylor instability, $1 / B$, is relevant for channels with aspect ratios $\alpha \geq 8$. In the case of square or near-square channels, our measurements confirm that the wet fraction is independent of the aspect ratio. For channels of arbitrary aspect ratios the wet fraction, a bulk measure of the flow, depends on a single parameter $\widehat{\mathrm{Ca}}=$ $\left[1+0.12(\alpha-1)+0.018(\alpha-1)^{2}\right] \mathrm{Ca}$, for values of $\widehat{\mathrm{Ca}}$ beyond a threshold value $\widehat{\mathrm{Ca}}_{t}$ that depends on buoyancy. For $\widehat{\mathrm{Ca}}<\widehat{\mathrm{Ca}}_{t}$ the scaling breaks down and the wet fraction tends to an asymptotic value that depends monotonically on $\alpha$. This novel scaling allows the determination of the wet fraction in any rectangular channel from the results in a square tube. From a fundamental standpoint this result establishes a quantitative connection between the bulk properties of interfacial flows in Hele-Shaw cells and square channels, despite significant local variations. The further understanding and prediction of the scaled parameter, transitional aspect ratio, $\alpha_{t}$, and threshold $\widehat{\mathrm{Ca}}_{t}$ pre- sented in this Letter offer a challenge to future theoretical studies.

The support of EPRSC is gratefully acknowledged.

[1] W. B. Kolb and R. L. Cerro, Chem. Eng. Sci. 46, 2181 (1991).

[2] A. Polynkin, J. F. T. Pittman, and J. Sienz, Chem. Eng. Sci. 60, 1591 (2005).

[3] J. B. Grotberg and O.E. Jensen, Annu. Rev. Fluid Mech. 36, 121 (2004).

[4] F. P. Bretherton, J. Fluid Mech. 10, 166 (1961).

[5] G. I. Taylor, J. Fluid Mech. 10, 161 (1961).

[6] L. W. Schwartz, H. M. Princen, and A. D. Kiss, J. Fluid Mech. 172, 259 (1986).

[7] V. S. Ajaev and G. M. Homsy, Annu. Rev. Fluid Mech. 38, 277 (2006).

[8] A. L. Hazel and M. Heil, J. Fluid Mech. 470, 91 (2002).

[9] P. G. Saffman and G. Taylor, Proc. R. Soc. A 245, 312 (1958).

[10] G. M. Homsy, Annu. Rev. Fluid Mech. 19, 271 (1987).

[11] C. Clanet, P. Héraud, and G. Searby, J. Fluid Mech. 519, 359 (2004).

[12] G. M. Whitesides and A. D. Stroock, Phys. Today 54, No. 6, 42 (2001).

[13] J. W. McLean and P. G. Saffman, J. Fluid Mech. 102, 455 (1981).

[14] P. Tabeling, G. Zocchi, and A. Libchaber, J. Fluid Mech. 177, 67 (1987).

[15] P. Tabeling and A. Libchaber, Phys. Rev. A 33, 794 (1986).

[16] M. G. Moore, A. Juel, J. M. Burgess, W. D. McCormick, and H. L. Swinney, Phys. Rev. E 65, 030601(R) (2002).

[17] H. Wong, C. J. Radke, and S. Morris, J. Fluid Mech. 292, 71 (1995).

[18] H. Wong, C. J. Radke, and S. Morris, J. Fluid Mech. 292, 95 (1995).

[19] The dynamic viscosities, surface tensions, and densities of the silicone oils were $\mu_{1}=0.934 \mathrm{cP}, \sigma_{1}=20.1 \mathrm{dyn} / \mathrm{cm}$, $\rho_{1}=934 \mathrm{~kg} / \mathrm{m}^{3} ; \quad \mu_{2}=4.815 \mathrm{cP}, \quad \sigma_{2}=20.8 \mathrm{dyn} / \mathrm{cm}$, $\rho_{2}=963 \mathrm{~kg} / \mathrm{m}^{3}$ and $\mu_{3}=97.4 \mathrm{cP}, \sigma_{3}=21.1 \mathrm{dyn} / \mathrm{cm}$, $\rho_{3}=974 \mathrm{~kg} / \mathrm{m}^{3}$ at $T=25^{\circ} \mathrm{C}$. The viscosities were measured for the temperature range of our experiments so that appropriate values were used in parameter calculations.

[20] V. Duclaux, C. Clanet, and D. Quéré, J. Fluid Mech. 556, 217 (2006).

[21] P. Aussillous and D. Quéré, Phys. Fluids 12, 2367 (2000).

[22] M. H. Jensen, A. Libchaber, P. Pelcé, and G. Zocchi, Phys. Rev. A 35, 2221 (1987).

[23] The errors indicate the parameter values at which the distance between the scaled curves exceeds the experimental error, provided that the other parameter remains fixed. The minimum is shallowest on the line $a_{2}=$ 0.0285-0.0875 $a_{1}$, however, along which $a_{1}$ can be reduced to 0.06 before experimental error is exceeded. 